\title{
Dibenzoylmethane ameliorates lipid-induced inflammation and oxidative injury in diabetic nephropathy
}

\author{
Eun Soo Lee1, Mi-Hye Kwon², Hong Min Kim¹, Nami Kim³, You Mi Kim¹, Hyeon Soo Kim³, Eun Young Lee ${ }^{4}$ and \\ Choon Hee Chung ${ }^{1}$
}

1Department of Internal Medicine, Yonsei University Wonju College of Medicine, Wonju, Korea

2The East Coast Research Institute of Life Science, Gangneung-Wonju National University, Gangneung, Korea

${ }^{3}$ Department of Anatomy, Korea University College of Medicine, Seoul, Korea

${ }^{4}$ Department of Internal Medicine and Institute of Tissue Regeneration, Soonchunhyang University, Cheonan, Korea

Correspondence should be addressed to C H Chung: cchung@yonsei.ac.kr

\begin{abstract}
Dibenzoylmethane (DBM) is a beta-diketone analog of curcumin. Numerous studies have shown the beneficial effects of curcumin on diabetes, obesity and diabetic complications including diabetic nephropathy. Recently, we investigated the beneficial metabolic effects of DBM on high-fat diet-induced obesity. However, the effects and mechanisms of action of DBM in the kidney are currently unknown. To investigate the renoprotective effects of DBM in type 2 diabetes, we administered DBM $(100 \mathrm{mg} / \mathrm{kg}$ ) orally for 12 weeks to high-fat diet-induced diabetic model mice. We used mouse renal mesangial (MES13) and macrophage (RAW 264.7) cells to examine the mechanism of action of DBM (20 $\mu \mathrm{M})$. After DBM treatment, the albumin-to-creatinine ratio was significantly decreased compared to that of the high-fat-diet group. Moreover, damaged renal ultra-structures and functions including increased glomerular volume, glomerular basement membrane thickness and inflammatory signals were ameliorated after DBM treatment. Stimulation of MES13 and RAW264.7 cells by palmitate or high-dose glucose with lipopolysaccharides increased inflammatory signals and macrophage migration. However, these changes were reversed by DBM treatment. In addition, DBM inhibited NADPH oxidase 2 and 4 expression and oxidative DNA damage. Collectively, these data suggested that DBM prevented diabetes-induced renal injury through its anti-inflammatory and antioxidant effects.
\end{abstract}

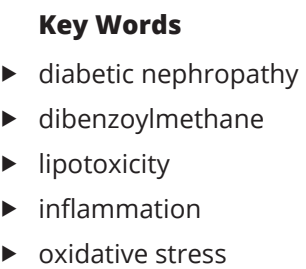

\section{Introduction}

The rapid onset of obesity is one of the main causes of insulin resistance and diabetic complications, such as retinopathy, neuropathy, cardiovascular disease and diabetic nephropathy (DN), which are associated with elevated glucotoxicity, lipotoxicity, inflammation and cellular apoptosis (Serrano Rios 1998). Among these complications, DN is a leading cause of end-stage kidney diseases and is associated with increased morbidity and mortality in diabetes. Under obesity-induced excessive cellular stress conditions, NADPH oxidase (NOX)-derived reactive oxygen species (ROS) are increased and accelerate macrophage polarization to proinflammatory macrophage M1 and activation. In particular, activated macrophages are recognized 
as prominent inflammatory cells that secrete many proinflammatory cytokines and chemokines, as well as pro-fibrotic and anti-angiogenic factors that contribute to the pathogenesis of DN. Continuous macrophages accumulation is correlated with proteinuria and renal fibrosis and is inversely correlated with renal function in humans (Nguyen et al. 2006). Thus, suppressing the infiltration of inflammatory macrophages alleviated insulin resistance and DN (Nistala et al. 2014, Eun Lee et al. 2016). Studies by Kodera et al. (2014) and Seok et al. (2013) showed that the administration of dipeptidyl peptidase 4 (DPP4) or C-C chemokine receptor type 2 (CCR2) inhibitors to diabetic animal models ameliorates DN through the inhibition of macrophage infiltration and inflammation (Seok et al. 2013, Kodera et al. 2014).

Curcumin, a polyphenolic compound derived from dietary turmeric, is known to have anti-inflammatory, antioxidant and antiangiogenic effects (Anand et al. 2007). In obesity or diabetes, curcumin improves insulin resistance and fatty liver by increasing fatty oxidation and decreasing lipid synthesis (Zhao et al. 2018). Furthermore, curcumin also ameliorates DN by inhibiting macrophage infiltration and inflammatory signaling including nuclear factor (NF)-kB p65 and antioxidant effects (Soetikno et al. 2011). Many studies have investigated the protective effects of curcumin and our previous study reported that curcumin ameliorates DN by regulating renal lipid metabolism and oxidative stress (Trujillo et al. 2013, Pan et al. 2013a, Kim et al. 2016, Lu et al. 2017). In addition to curcumin its analogs such as C66 and B06 were also identified to have $\mathrm{DN}$ therapeutic effects by the inhibition of inflammation and macrophage infiltration (Pan et al. 2013b, Wu et al. 2016).

Dibenzoylmethane (DBM), a beta-diketone analog of curcumin, has been reported to have anticarcinoma (Khor et al. 2009), antioxidant and cellular protective effects against endoplasmic reticulum stress (Takano et al. 2007). Moreover, our previous study showed the beneficial metabolic effects of DBM, which regulates glucose uptake via AMP-activated protein kinase (AMPK) activation in skeletal muscle and inhibits fatty acid synthesis in pre-adipocytes (Kim et al. 2015). However, the effects and action mechanisms of DBM on DN have not yet been studied.

Using a high-fat diet (HFD)-induced obesity mouse model, we sought to investigate the potential protective effects of DBM against DN and clarify its mechanisms of action.

\section{Materials and methods}

\section{Animal study}

Four-week-old male C57BL/6 mice (15g) were purchased from Dae Han Bio Link Co. (Chungbuk, Korea). The animal experiments were conducted as described in a previous study (Kim et al. 2015). In brief, animals were housed in cages placed in a room on a 12-h light/darkness cycle and ambient temperature $\left(22-24^{\circ} \mathrm{C}\right)$. At 8 weeks, the mice were randomly divided into the following three groups of ten each: (i) normal diet fed (ND), (ii) HFD fed (HFD, 60\% kcal from fat) and (iii) DBM-treated (HFD+DBM $100 \mathrm{mg} / \mathrm{kg}$ ) groups. DBM, an analog of curcumin (Fig. 1), was administered by mixing with the chow. Food intake and body weights were recorded weekly. After 12 weeks, the animals were anesthetized, and the serum and tissues were collected and stored for further studies. All experiments were approved by the Institutional Animal Care and Use Committee of Yonsei University at the Wonju Campus (YWC-121030-1).

\section{Assessment of urine albumin and creatinine}

Urine was collected using a metabolic cage for $24 \mathrm{~h}$ and the urinary albumin and creatinine levels were measured using the Albuwell $\mathrm{M}$ and Creatinine Companion Kits in accordance with the manufacturer's instructions (Exocell, Inc., Philadelphia, PA, USA).<smiles>COc1cc(/C=C/C(=O)/C=C(O)/C=C/c2ccc(O)c(OC)c2)ccc1O</smiles>

Curcumin

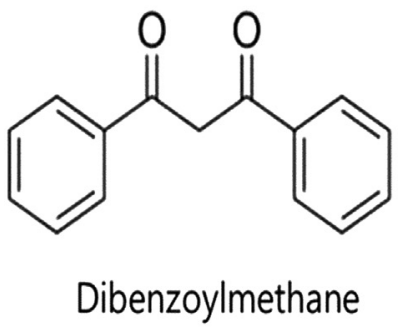

Figure 1

Chemical structures of curcumin and dibenzoylmethane (DBM). 


\section{Measurement of renal triglycerides (TGs)}

The renal TG content was examined in kidney tissues. Briefly, ethanolic potassium hydroxide was added to the tissue and the mixture was incubated at $55^{\circ} \mathrm{C}$ overnight. Then, $50 \%$ ethanol was added to the tube, which was centrifuged for $5 \mathrm{~min}$. The supernatant was transferred to a new tube, $50 \%$ ethanol was added and the mixture was vortexed. The supernatant was transferred to a new tube, $1 \mathrm{M}$ magnesium chloride was added and then the mixture was kept on ice for $10 \mathrm{~min}$. The tube was centrifuged for $5 \mathrm{~min}$ and the supernatant was transferred to a new tube. To determine the glycerol content, reconstituted glycerol reagent (Sigma-Aldrich) was added according to the kit instructions. The mixture was incubated for $15 \mathrm{~min}$ and the absorbance was measured at $540 \mathrm{~nm}$. Renal TG levels were calculated using the following formula: TG $(\mathrm{mg} / \mathrm{g}$ tissue $)=$ cuvette triolein equivalent glycerol concentration $(\mathrm{mg} / \mathrm{dL}) \times(10 / 30) \times(415 / 200) \times 0.012$ (dL)/tissue weight (g).

\section{Transmission electron microscopy}

Slit pore numbers and the glomerular basement membrane (GBM) thickness were measured using a transmission electron microscope (JEM-1200EX II, JEOL, Ltd, Tokyo, Japan). Electron micrographs were captured at 20,000x magnification for each sample.

\section{Estimation of glomerular volume and adipocyte size}

Paraffin-embedded kidney and epididymal fat tissues were stained with hematoxylin and eosin (H\&E). The tissue sections were examined using an optical microscope equipped with a charge-coupled device camera (Pulnix, Sunnyvale, CA, USA).

\section{Immunohistochemistry of glomeruli}

Paraffin-embedded kidney tissues were stained for monocyte chemoattractant protein-1 (MCP1), inflammatory macrophage markers (F4/80 and CD11c), and oxidative stress marker (8-hydroxy-2'-deoxyguanosine (8-OHdG) ) using a commercial immunohistochemistry staining kit in accordance with the manufacturer's instructions (Thermo Scientific). Positively stained area and cells were counted with ImageJ program (National Institute of Mental Health, Bethesda, MD, USA).

\section{RNA extraction and quantitative real-time polymerase chain reaction (qPCR)}

Total RNA from the kidney was isolated using TRIzol (Sigma-Aldrich) according to a standard protocol. cDNA was prepared from $1.0 \mu \mathrm{g}$ total RNA of each sample using a commercially available kit (QuantiTect Reverse Transcription Kit; Promega, Hilden, Germany). qPCR was performed using a SYBR Green PCR master mix (Applied Biosystems) using an ABI PRISM 7900HT sequence detection system (Applied Biosystems). The following primers were used: mMcp1: sense, 5'-CTT GCC TAA TCC ACA GAC TG-3' and antisense, 5'-GCC TGA ACA GCA CCA CTA-3'; interleukin (IL)-1 1 : sense, 5'-GGG CTG CTT CCA AAC CTT TG-3' and antisense, $5^{\prime}$-GGG CTG CTT CCA AAC CTT TG-3'; mNox2: sense, 5'-AAC TCC GCT CTT TCA CCA GG-3' and antisense, 5'-GTG i ACA CCC AGA GTC AG-3'; mNox4: sense, 5'-TGT TGG GCC TAG GAT TGT GTT-3' and antisense, 5'-GGC TAC ATG CAC ACC TGA GA-3' and glyceraldehyde 3-phosphate dehydrogenase (GAPDH): sense, 5'-CTG GAG AAA CCT GCC AAG TA-3' and antisense 5-AGT GGG AGT TGC TGT TGA AG-3'.

\section{Western blot analysis}

Kidney tissues and cultured cells were homogenized in the PRO-PREP protein extraction solution (iNtRON Biotechnology, Korea) containing proteinase and phosphatase inhibitors (GenDEPOT, Barker, TX, USA). The protein concentration in the supernatant was measured using a bicinchoninic acid protein assay kit (Pierce). The protein samples were electrophoresed on $8-12 \%$ SDS-PAGE and the resolved proteins were transferred to PVDF membranes. The blots were incubated with the following primary antibodies: anti-arginase 2, anti-cluster of differentiation 68 (CD68), anti-nuclear factor, erythroid 2 like 2 (NRF2), anti-heme oxygenase1 (HO1), anti-NOX2, and anti-NOX4 (Santa Cruz Biotechnology), anti- $\beta$-actin, anti-nephrin (Abcam), anti-phospho (p)-AMPK, antip-P38, anti-p38, anti-p-inhibitor of NF-kB (IkB) kinase (IKK) $\beta$, anti-IKK $\beta$, anti-IkB, anti-p-NFkB p65 and antiNFkB p65 (Cell Signaling Technology) antibodies. The blots were visualized using a chemiluminescence UVP BioSpectrum 600 imaging system and quantified with ImageJ program (National Institute of Mental Health).

\section{Cell culture}

Mouse mesangial (MES-13) cells were cultured at $37.5^{\circ} \mathrm{C}$ in Dulbecco's modified Eagle's medium (DMEM) 
containing $5.5 \mathrm{mM}$ glucose, $1 \%$ antibiotics and $10 \%$ fetal bovine serum (FBS). The cultured cells were starved for $24 \mathrm{~h}$ and treated with low glucose $(5.5 \mathrm{mM})$ and palmitate $(\mathrm{PA}, 250 \mu \mathrm{M})$ with or without DBM $(20 \mu \mathrm{M})$. Mouse macrophages Raw 264.7 cells were cultured at $37.5^{\circ} \mathrm{C}$ in DMEM containing $25.5 \mathrm{mM}$ glucose, $1 \%$ antibiotics and $5 \%$ FBS. To prepare the conditioned medium (CM), Raw 264.7 cells were stimulated with lipopolysaccharide (LPS, $100 \mathrm{ng} / \mathrm{mL})$, in the presence or absence of DBM $(20 \mu \mathrm{M})$ for $24 \mathrm{~h}$. The collected $\mathrm{CM}$ was stored at $-80^{\circ} \mathrm{C}$ until further use.

\section{Migration assay}

To determine whether DBM inhibited the migration of RAW 264.7 cells, an LPS stimulation migration assay was performed in a 96-well chemotaxis chamber (ChemoTx; Neuro Probe, Gaithersburg, MD, USA). The lower chambers were filled with $30 \mu \mathrm{L}$ medium in the presence or absence of LPS or DBM. Cells $\left(1.4 \times 10^{4} / 50 \mu \mathrm{L}\right)$ were seeded in the upper chambers and incubated for $4 \mathrm{~h}$ at $37^{\circ} \mathrm{C}$ in an atmosphere of $5 \% \mathrm{CO}_{2}$. Cells that had migrated to the bottom side were fixed for $5 \mathrm{~min}$ in methanol and the nuclei were stained with hematoxylin for $5 \mathrm{~min}$. The cytoplasm was stained with an eosin solution for $1 \mathrm{~min}$, and the cells were washed with tap water to remove the background stain. The stained cells were then photographed and counted using an image analysis system (Olympus SIS).

\section{NOX2 and NOX4 immunofluorescence staining}

MES-13 cells were seeded into chamber slides and incubated in starvation medium for $12 \mathrm{~h}$ and the cells were transferred to high-glucose (HG, $30 \mathrm{mM}$ ) medium with LPS in the presence or absence of DBM. After $24 \mathrm{~h}$, the culture medium was removed and the cells were fixed in $4 \%$ paraformaldehyde solution for $15 \mathrm{~min}$ at room temperature. Then, the cells were stained for NOX2 and NOX4 using a commercial immunohistochemistry kit in accordance with the manufacturer's instructions (Thermo Scientific).

\section{Statistics}

All data are expressed as the means \pm standard error of the mean. A Student's t-test was used for experiments with only two groups. Differences were considered statistically significant when $P<0.05$.
A

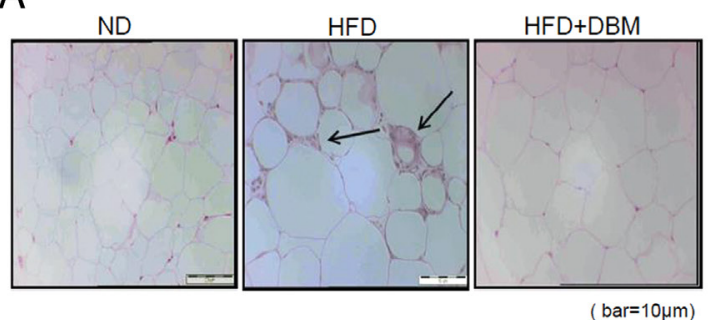

B

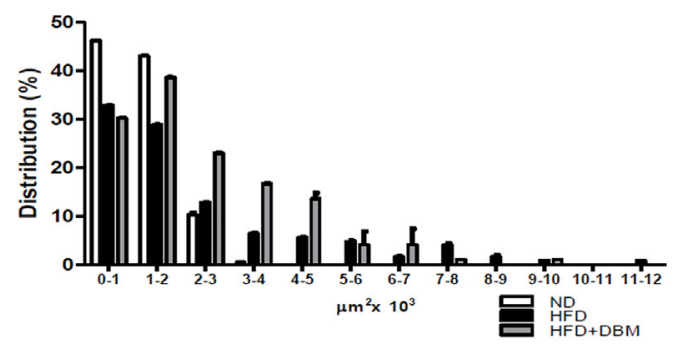

C

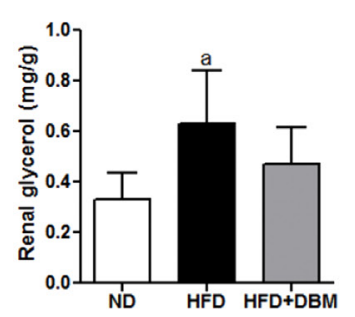

D

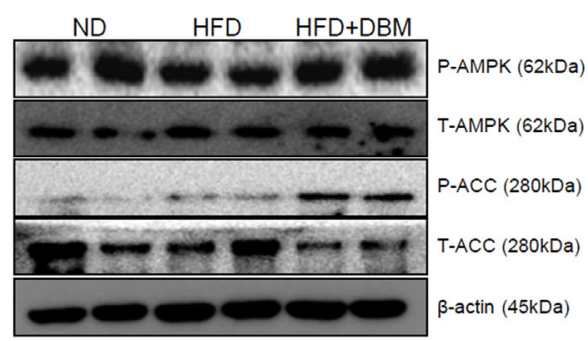

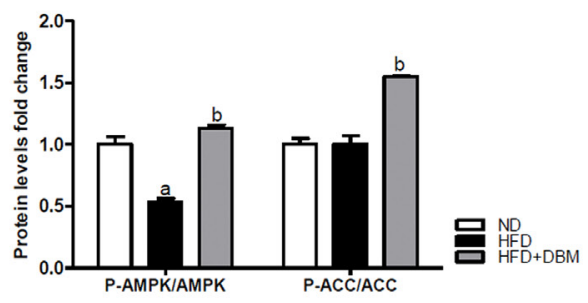

\section{Figure 2}

Changes in number of high-fat diet (HFD)-induced crown-like structures in white adipose tissue and levels of renal triglycerides caused by dibenzoylmethane (DBM) administration. (A) H\&E staining of white adipose tissue. (B) Measurement of adipocyte sizes. (C) Renal TG levels were measured by converting TG to glycerol. (D) Renal p-AMPK and p-ACC levels were analyzed using western blotting. Arrow, crown-like structures; DBM, HFD plus dibenzoylmethane; H\&E, hematoxylin and eosin; HFD, high-fat diet group; ND, normal diet group; TG, triglycerides; WAT, white adipose tissue. a $P<0.05$ compared with the ND group; $\mathrm{b} P<0.05$ compared with HFD group. A full colour version of this figure is available at https://doi. org/10.1530/JOE-18-0206. 


\section{Results}

\section{DBM reduces adipocyte size and number of crown-like structures in epididymal fat}

The HFD-induced diabetic mouse model of obesity exhibits adipocyte hypertrophy and crown-like structures in fat tissues (Sun et al. 2011). In the HFD group, crown-like structure formation and adipocyte size were increased compared with that of the ND group (Fig. 2A and $\mathrm{B})$. However, these changes were reversed by DBM administration.

\section{DBM inhibits renal fatty acid synthesis by activating AMPK}

A high level of TG is a risk factor for the progression of renal disease in humans (Trevisan et al. 2006). To measure the levels in the kidneys, TG was hydrolyzed to glycerol. HFD increased renal glycerol levels, but DBM administration to the HFD group did not significantly increase the levels compared with those of the ND group (ND, $0.33 \pm 0.1$; HFD, $0.63 \pm 0.21$, and HFD+DBM; $0.47 \pm 0.15 \mathrm{mg} / \mathrm{g}$, Fig. 2C). In the HFD group, renal p-AMPK levels were significantly decreased, but acetyl-CoA carboxylase
(ACC) phosphorylation did not change, whereas DBM treatment activated AMPK and ACC phosphorylation (Fig. 2D). These findings suggest that DBM regulates renal lipid metabolism.

\section{DBM improves damaged renal structure and urinary albumin-to-creatinine ratio (ACR)}

The urinary ACR is a marker of impaired renal function and the level sharply increased in the HFD group, but DBM reversed the decreased urinary ACR (Fig. 3A). Moreover, the renal glomerular volume increased in the HFD group, but was similarly restored by DBM (Fig. 3B and C). Structurally, increased renal GBM thickness, podocyte effacement and slit diaphragm loss occurred in the HFD group, but these changes were alleviated by DBM (Fig. 3D, E and F). These data indicate that DBM exerts protective effects against renal injury induced by diabetes.

\section{DBM inhibits macrophage infiltration and inflammation in DN}

Using western blotting assays, we determined that the expression of renal nephrin protein was lower in
A

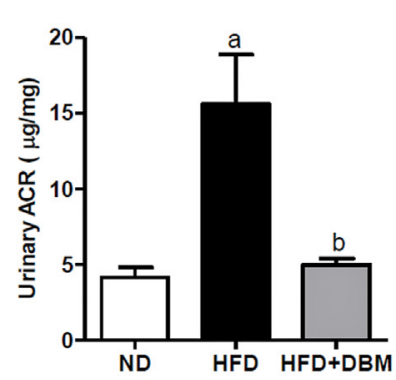

B

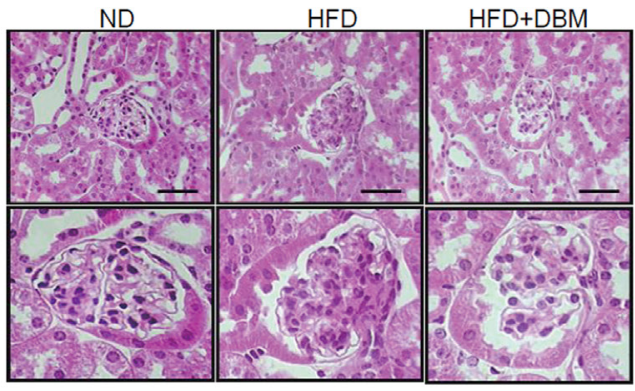

E

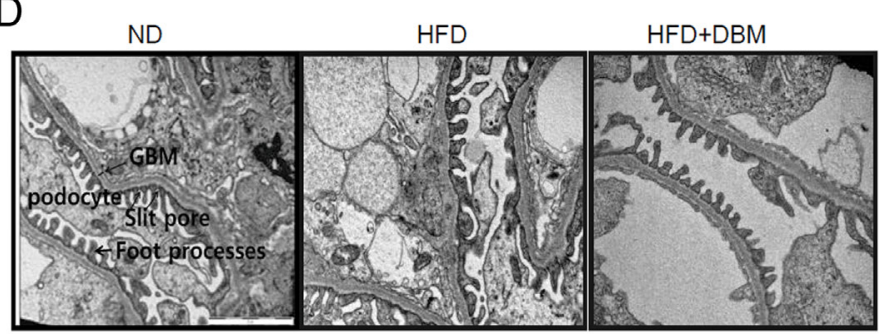

C

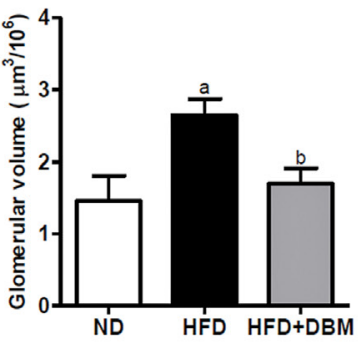

$\mathrm{F}$

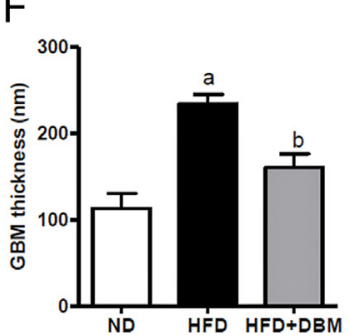

Figure 3

Effects of dibenzoylmethane (DBM) on high-fat diet (HFD)-induced diabetic nephropathy (DN). (A) Albumin-to-creatinine ratio (ACR) was measured in urine over a 24-h collection period. (B) Glomerular expansion was analyzed using H\&E staining of renal glomeruli and renal ultrastructure was analyzed using transmission electron microscopy (TEM). Changes in glomerular volumes (C), slit pore numbers (D and E) and GBM thickness (D and F) were determined. ACR, albumin/creatinine ratio; DBM, HFD plus dibenzoylmethane; GBM, glomerular basement membrane; HFD, high-fat diet group; ND, normal diet group. a $P<0.05$ compared with the ND group; ${ }^{b} P<0.05$ compared with HFD group. A full colour version of this figure is available at https:// doi.org/10.1530/JOE-18-0206. 
A

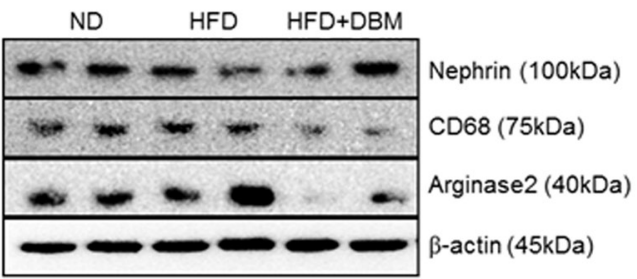

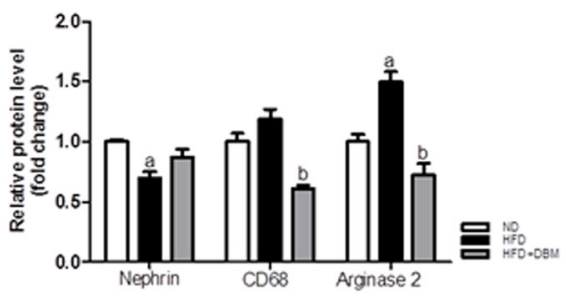

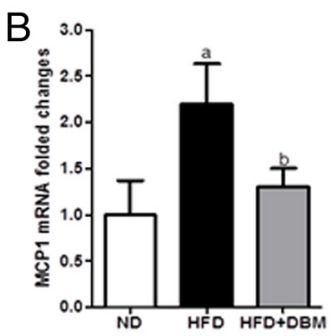

D

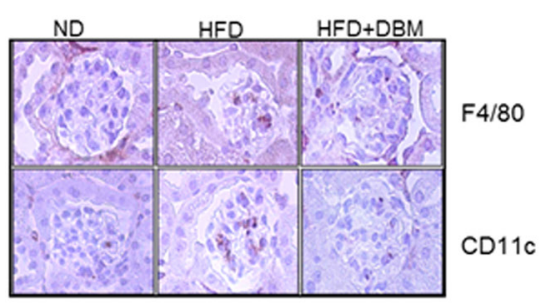

E

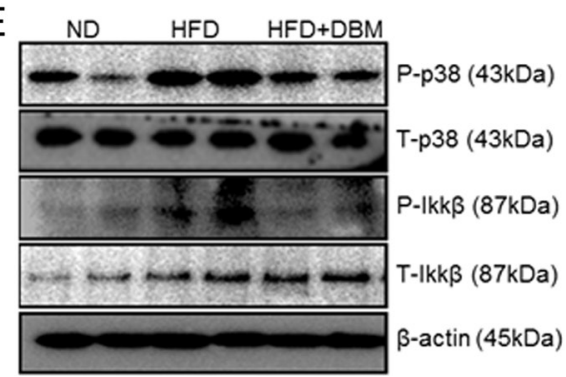

C
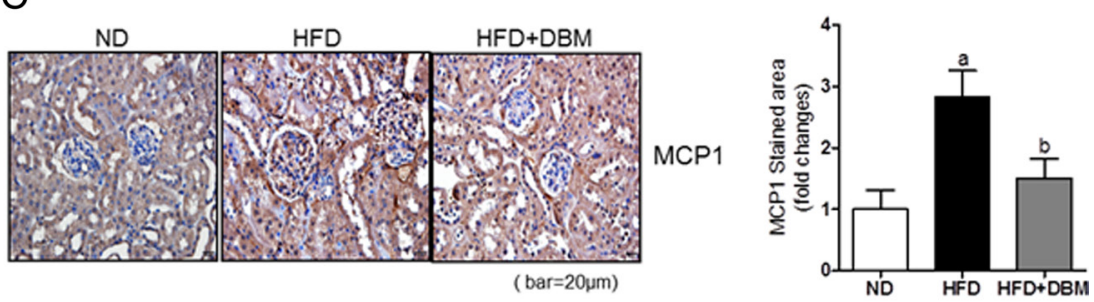
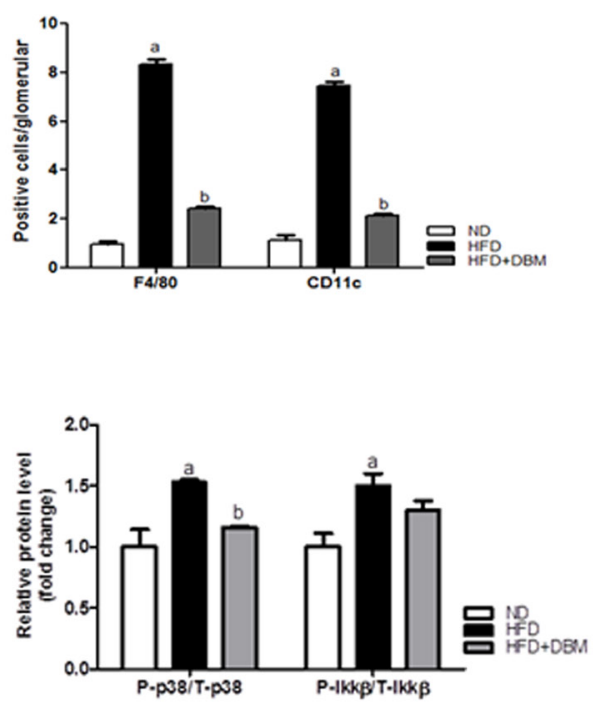

\section{Figure 4}

Effects of dibenzoylmethane (DBM) on macrophage infiltration and inflammatory signals in the kidney. Renal nephrin, CD68, and arginase 2 levels were analyzed using western blotting and quantified (A). Renal MCP1 mRNA level was quantified using qPCR (B). Immunohistochemical staining of the MCP1 (C), F4/80 and CD11c (D). p-AMPK and inflammatory signals, including p-p38 and p-IKK $\beta$, were detected by western blotting in the kidney and quantified (E). AMPK, AMP-activated protein kinase; DBM, HFD plus dibenzoylmethane; HFD, high-fat diet group; ND, normal diet group; qPCR, quantitative polymerase chain reaction. a $P<0.05$ compared with the ND group; $b P<0.05$ compared with HFD group. A full colour version of this figure is available at https://doi.org/10.1530/JOE-18-0206.

the HFD group than in the ND group. DBM restored the level of nephrin expression. In the DBM group, both the protein levels of HFD-induced inflammation-related genes such as CD68 and arginase 2 and the mRNA levels of inflammatory cytokines such as MCP1 were significantly decreased by DBM (Fig. 4A and B). Moreover, $\mathrm{MCP} 1$, total macrophages (F4/80) and inflammatory macrophage (CD11c) markers were also strongly stained in the glomeruli of the HFD group compared to those of the ND group, but these effects were reduced by DBM administration (Fig. 4C and D). Furthermore, increased p-p38 and p-IKK kinase $\beta$ expression levels in the HFD group were significantly decreased by DBM administration (Fig. 4E). These findings suggest that DBM suppressed macrophage infiltration to the glomeruli and inflammation.

\section{DBM activates AMPK more than curcumin in MES-13 cells}

DBM activation of AMPK was stronger than that of curcumin, and AMPK phosphorylation increased in a dose-dependent manner in MES-13 cells (Fig. 5A and B). The HG-induced reduction of AMPK phosphorylation was 
restored by DBM treatment. This change was inhibited by the AMPK inhibitor compound C (Fig. 5C). Thus, these findings suggested that DBM was a more potent AMPK activator than curcumin.

\section{DBM inhibits inflammatory signal activation and macrophage migration}

PA stimulation activated inflammatory signals, such as IкB degradation and NFkB p65 phosphorylation, which were ameliorated by DBM treatment (Fig. 5D). Moreover, DBM treatment with LPS suppressed RAW264.7 cell migration (Fig. 5E). In the CM, IKK activation, NFкB p65 phosphorylation and IкB degradation were observed in the renal proximal tubule cells, but these effects were also ameliorated by DBM treatment (Fig. 5F). These data indicated that DBM inhibited macrophage activity and inflammation.

\section{DBM exerts antioxidant effects on DN}

In mesangial cells, HG and LPS stimulation increased the expression of both NOX2 and NOX4. With these changes, antioxidant pathways such as Nrf2/HO-1 expression were also activated. However, DBM treatment reduced these changes (Fig. 6A, B and C) and inhibited HFD-induced 8-OHdG expression in the glomeruli (Fig. 6D). These findings suggested that DBM exerted renoprotective effects through antioxidant activity.
A

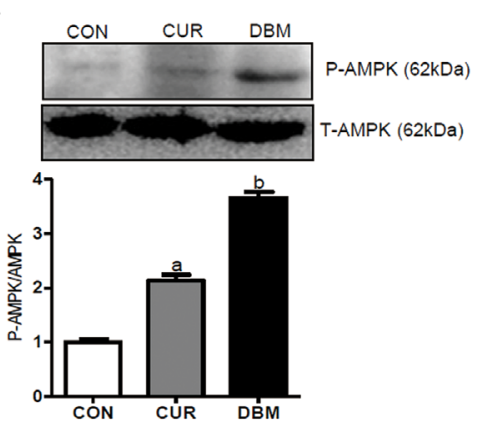

D $\mathrm{PA}$ - - + + DBM - +-+

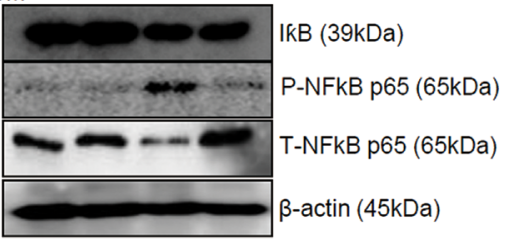

E

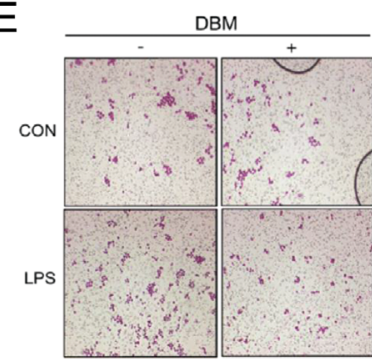

B
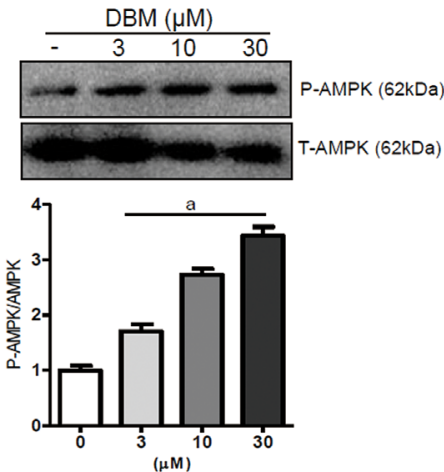
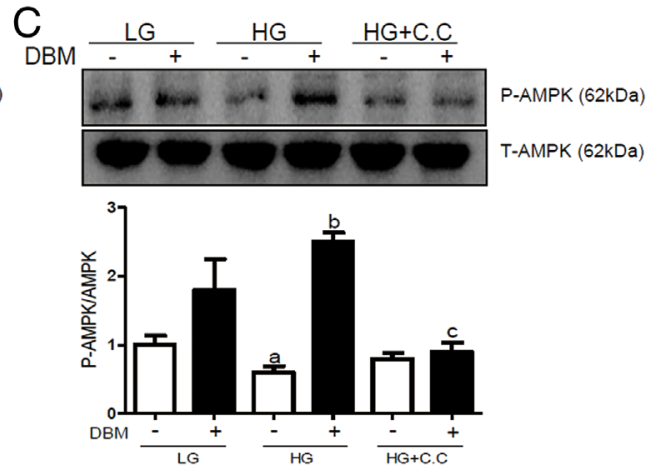

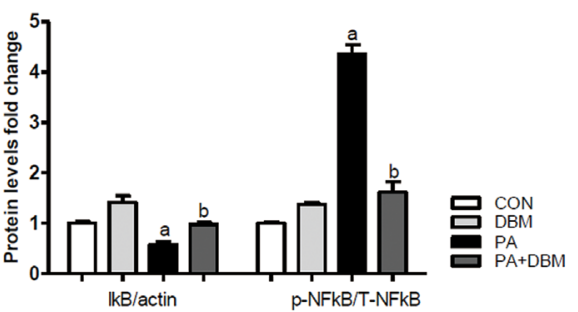

$\mathrm{F}$
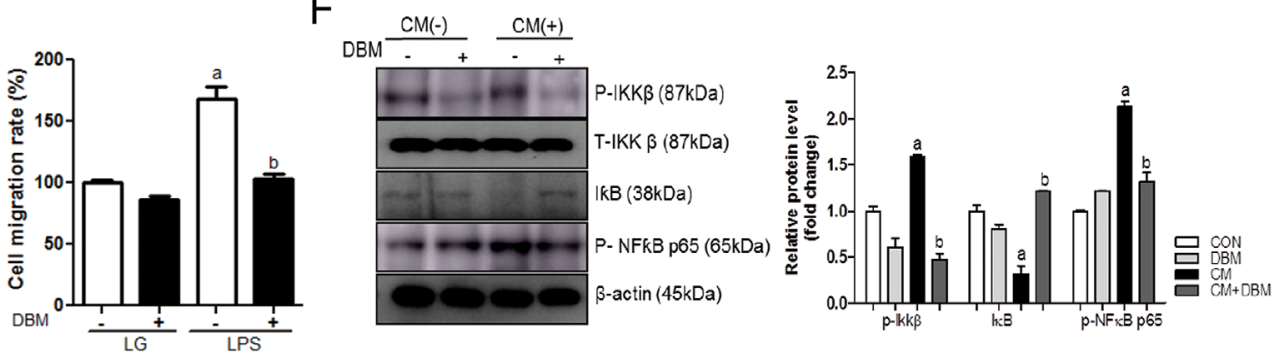

Figure $\mathbf{5}$

AMP-activated protein kinase (AMPK) activation by dibenzoylmethane (DBM) and curcumin. (A) Comparison of effects of curcumin and DBM on AMPK activation in NRK-52E cells. (B) DBM dose-dependently activated p-AMPK. (C) Changes in p-AMPK expression induced by high-glucose (HG), compound C, or both in the presence or absence of DBM. (D) PA-induced inflammatory signals (IkB and p-NFkB p65) in MES-13 cells caused by DBM treatment. (E) LPS-induced macrophage migration was affected by DBM treatment. (F) p-IKK, IkB, and p-NFkB p65 levels were changed by CM and changes were reversed by DBM treatment. CM, Raw 264.7 cultured medium with LPS; DBM, dibenzoylmethane; HG, high-glucose, 30 mM glucose in DMEM; LG, low-glucose, 5.5 mM glucose in DMEM; LPS, lipopolysaccharide; PA, palmitate. a $P<0.05$ compared with LG; bP < 0.05 compared with HG. A full colour version of this figure is available at https://doi.org/10.1530/JOE-18-0206. 


\begin{tabular}{l|l|l|r|r|}
\hline $\begin{array}{l}\text { Journal of } \\
\text { Endocrinology }\end{array}$ & E S Lee et al. & $\begin{array}{l}\text { Dibenzoylmethane in diabetic } \\
\text { nephropathy }\end{array}$ & $\mathbf{2 4 0 : 2}$ & $\mathbf{1 7 6}$ \\
\hline
\end{tabular}
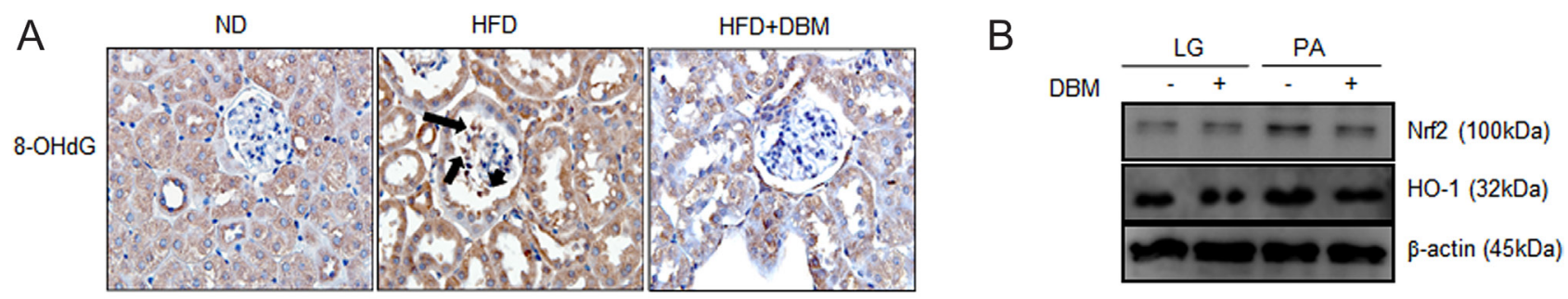

C

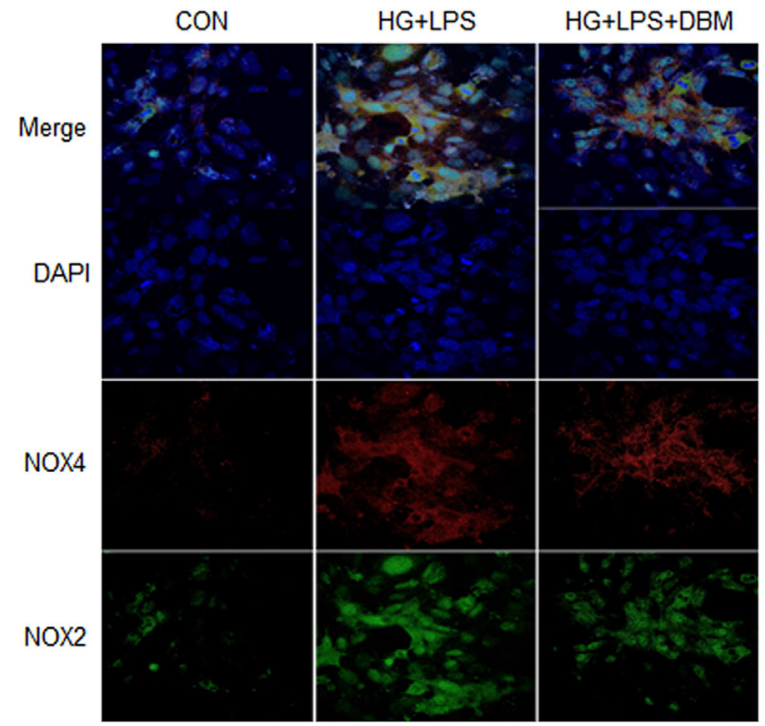

D
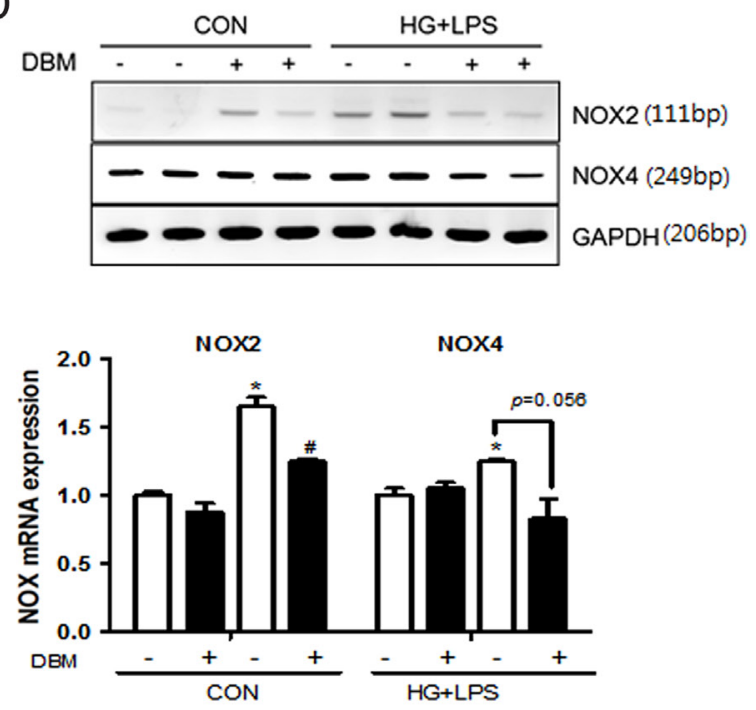

\section{Figure 6}

Antioxidant effects of dibenzoylmethane (DBM). (A) Oxidative DNA damage marker, 8-OHdG, showed staining in glomeruli. (B) Changes in Nrf2/HO-1 expression by DBM in HG and LPS-stimulated MES-13 cells. (C and D) NOX2 and NOX4 protein and mRNA expressions change by DBM treatment in HG with LPS-stimulated MES-13. 8-OHdG, 8-hydroxy-2'-deoxyguanosine; DBM, dibenzoylmethane; HG, high-glucose, 30 mM glucose in DMEM; LG, lowglucose, $5.5 \mathrm{mM}$ glucose in DMEM; LPS, lipopolysaccharide. a $P<0.05$ compared with LG; $\mathrm{b} P<0.05$ compared with HG. A full colour version of this figure is available at https://doi.org/10.1530/JOE-18-0206.

\section{Discussion}

Obesity induces cellular stress concomitantly with metabolic syndromes, including insulin resistance, glucose intolerance, dyslipidemia and hypertension, as well as the induction of DN by increased cellular inflammation mediated by the interaction between oxidative stress induction and macrophage interaction, which exacerbates cellular damage to lipids, proteins and DNA (Keaney et al. 2003, Qatanani \& Lazar 2007). Metabolic inflammation is intensified in obesity by the infiltration of activated macrophages into tissues and secretion of inflammatory cytokines such as tumor necrosis factor (TNF)- $\alpha$, IL-1 $\beta$ and IL-6 (McArdle et al. 2013).

NOXs have been regarded as the major contributors to ROS generation. They consist of seven subunits, NOX1 to 5 and dual oxidase 1 (DUOX1) and 2. Among them, NOX2 and 4 are predominantly expressed in DN (Sedeek et al. 2010, You et al. 2013). Excessive ROS generation accelerates macrophage infiltration, and numerous studies have provided strong evidence that macrophages are the cause of renal disease. Macrophage infiltration into the kidney in diabetes, which is a characteristic feature of $\mathrm{DN}$, is one of the central mediators of renal vascular inflammation (Sassy-Prigent et al. 2000). MCP1 or chemokine (C-C motif) ligand 2 (CCL2) is one of the key chemokines that regulates the infiltration of monocytes/macrophages (Deshmane et al. 2009). Moreover, urinary MCP1/CCL2 levels are significantly increased in patients with $\mathrm{DN}$ and are correlated with the infiltration of CD68-positive macrophages into the renal interstitium. NFkB signaling, activated by obesity, also increases proinflammatory chemokine production in macrophages (Mezzano et al. 2004, Eardley et al. 2006). In our previous study, the blockade of the MCP1 receptor by a CCR2 inhibitor ameliorated DN by preventing macrophage infiltration and inflammation in type 2 diabetic mice (Seok et al. 2013). Based on these data and the suppression of oxidative stress, the inhibition of inflammation is also considered to be an effective target 
for DN treatment. In this study, DBM treatment reduced both macrophages infiltration and MCP1 expression, resulting in reduced oxidative-induced DNA damage of the glomeruli.

AMPK is a ubiquitous heterotrimeric kinase, which consists of a catalytic $\alpha$-subunit and regulatory $\beta$ - and $\gamma$-subunits. The enzyme, which is highly expressed in the kidneys, has various physiological functions and is involved in pathological processes, including ion transport, podocyte function and diabetic renal hypertrophy (Hallows et al. 2010). In diabetes, increase in numerous intracellular factors such as glucose, vascular endothelial growth factor MAPJ and angiotensin II lead to the inhibition of AMPK activation and induction of DN. Moreover, reduced AMPK activation associated with autophagy deficiency and cellular apoptosis subsequently induces diabetic complications including renal injury (Yao et al. 2016). Infiltrating macrophages secrete inflammatory cytokines, such as TNF- $\alpha$, and induce apoptosis of proximal tubule cells (Wang et al. 2015). Conversely, an AMPK activator was shown to inhibit the proinflammatory responses by decreasing inflammatory stimuli induced by cellular ROS production and mitogenactivated protein kinase activation in macrophages (Jeong et al. 2009).

Curcumin, the active ingredient of turmeric, has been extensively investigated for its beneficial effects on health such as anticancer (Zhang et al. 2015), antioxidant (Xiong et al. 2015), antidiabetic (He et al. 2012) and antiinflammatory in HFD obese rats (Maithilikarpagaselvi et al. 2016), which were shown to be mediated by AMPK activation. The renoprotective effects of curcumin have already been investigated in many studies (Soetikno et al. 2013, Ho et al. 2016). Our previous study also reported that curcumin ameliorates DN in type 2 diabetic rats through its antioxidant effects and regulation of renal lipid metabolism (Kim et al. 2016).

DBM, a $\beta$-diketone structural analog of curcumin (Fig. 1), has also received much attention owing to its biological properties. DBM has been reported to show anti-inflammatory effects (Anand et al. 2011), cell cycle regulation (Jackson et al. 2002) and anti-mutagenic effects (Shishu et al. 2003). We previously demonstrated the beneficial metabolic effects of DBM; in summary, DBM increased the phosphorylation of AMPK, stimulated glucose uptake in skeletal muscle cells and reduced the expression of fatty acid synthesis-related genes in preadipocytes. Moreover, DBM suppressed HFD-induced weight gain and fat accumulation in the liver (Kim et al. 2015). Although DBM exhibits antidiabetic effects, its beneficial effects on DN have not been studied. Therefore, we studied the effects of DBM on DN and found that the compound reduced the number of HFD-induced crown-like structures, which consisted of dead or dying adipocytes surrounded by macrophages, in white adipose tissue. To evaluate the effect of DBM on lipid metabolism, we determined a renal TG level by hydrolyzing TGs to glycerol. The renal glycerol level was significantly increased by HFD, whereas DBM administration slightly reduced this level; however, the differences were not significant. The infiltration of inflammatory macrophage into the renal glomeruli and inflammatory signals, which were increased by HFD, were inhibited by DBM administration.

In this study, both in vivo and in vitro experiments indicated that renal AMPK activation decreased in the HFD group, whereas inflammatory signals such as p38, c-Jun N-terminal kinase, and IKK were increased. LPS stimulation increased Raw 264.7 cell migration and Raw 264.7 CM-induced inflammatory signals in NRK-52E cells; however, these changes were alleviated by DBM treatment. A study by Miller et al. (2008) showed that the macrophage migration inhibitory factor modulated AMPK activation, as well as functionally linked inflammation and metabolism in the heart (Miller et al. 2008) and induced cardiomyocyte apoptosis. Moreover, the administration of metformin, an AMPK activator, suppressed the LPS-induced inflammatory response of Raw 264.7 cells (Kim et al. 2014). However, we did not investigate the mechanisms of DBM action on macrophages.

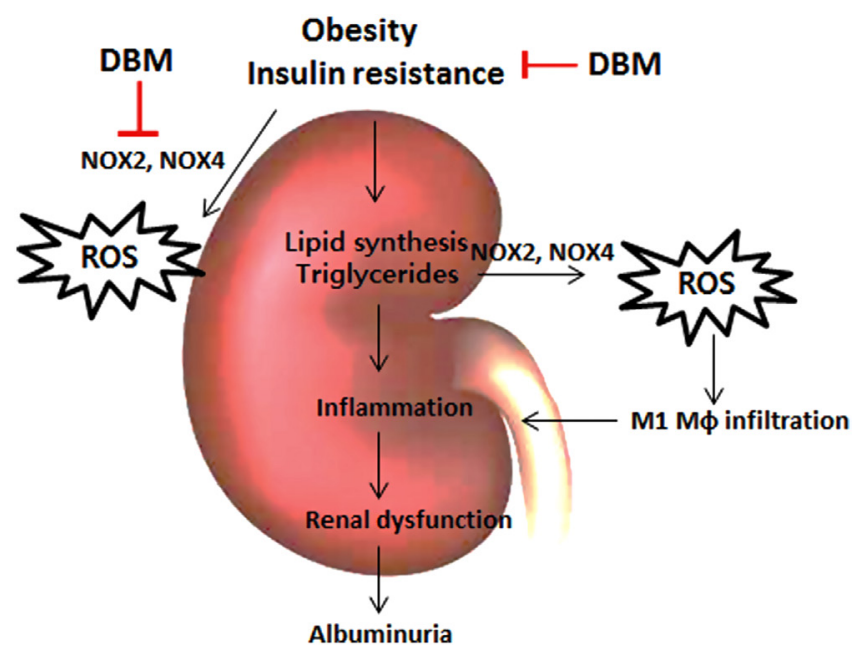

Figure 7

Schematic presentation of effects of dibenzoylmethane (DBM) on diabetic nephropathy (DN) in mouse model of high-fat diet (HFD)-induced diabetes. A full colour version of this figure is available at https://doi. org/10.1530/JOE-18-0206. 
Therefore, further studies are needed to elucidate how DBM directly regulates macrophage activation.

Collectively, as indicated in Fig. 7, the current study demonstrated the renoprotective effects of DBM against HFD-induced renal injury, which were mediated by the reduction of oxidative stress, attenuation of inflammatory macrophage infiltration into the kidneys, suppression of inflammatory signaling and stimulation of AMPK activation (Fig. 7). Therefore, DBM is a promising agent for the treatment of $\mathrm{DN}$.

\section{Declaration of interest}

The authors declare that there is no conflict of interest that could be perceived as prejudicing the impartiality of the research reported.

\section{Funding}

This work did not receive any specific grant from any funding agency in the public, commercial, or not-for-profit sector.

\section{Acknowledgements}

This research was supported by the Basic Science Research Program through the National Research Foundation of Korea (NRF) funded by the Ministry of Education (NRF-2016R1A6A3A11933581).

\section{References}

Anand P, Kunnumakkara AB, Newman RA \& Aggarwal BB 2007 Bioavailability of curcumin: problems and promises. Molecular Pharmaceutics 4 807-818. (https://doi.org/10.1021/mp700113r)

Anand P, Sung B, Kunnumakkara AB, Rajasekharan KN \& Aggarwal BB 2011 Suppression of pro-inflammatory and proliferative pathways by diferuloylmethane (curcumin) and its analoguesdibenzoylmethane, dibenzoylpropane, and dibenzylideneacetone: role of Michael acceptors and Michael donors. Biochemical Pharmacology 82 1901-1909. (https://doi.org/10.1016/i.bcp.2011.09.001)

Deshmane SL, Kremlev S, Amini S \& Sawaya BE 2009 Monocyte chemoattractant protein-1 (MCP-1): an overview. Journal of Interferon and Cytokine Research 29 313-326. (https://doi.org/10.1089/ jir.2008.0027)

Eardley KS, Zehnder D, Quinkler M, Lepenies J, Bates RL, Savage CO, Howie AJ, Adu D \& Cockwell P 2006 The relationship between albuminuria, MCP-1/CCL2, and interstitial macrophages in chronic kidney disease. Kidney International 69 1189-1197. (https://doi. org/10.1038/sj.ki.5000212)

Eun Lee J, Kim JE, Lee MH, Song HK, Ghee JY, Kang YS, Min HS, Kim HW, Cha JJ, Han JY, et al. 2016 DA-1229, a dipeptidyl peptidase IV inhibitor, protects against renal injury by preventing podocyte damage in an animal model of progressive renal injury. Laboratory Investigation 96 547-560. (https://doi.org/10.1038/labinvest.2016.34)

Hallows KR, Mount PF, Pastor-Soler NM \& Power DA 2010 Role of the energy sensor AMP-activated protein kinase in renal physiology and disease. American Journal of Physiology-Renal Physiology 298 F1067-F1077. (https://doi.org/10.1152/ajprenal.00005.2010)
He HJ, Wang GY, Gao Y, Ling WH, Yu ZW \& Jin TR 2012 Curcumin attenuates Nrf2 signaling defect, oxidative stress in muscle and glucose intolerance in high fat diet-fed mice. World Journal of Diabetes 3 94-104. (https://doi.org/10.4239/wjd.v3.i5.94)

Ho C, Hsu YC, Lei CC, Mau SC, Shih YH \& Lin CL 2016 Curcumin rescues diabetic renal fibrosis by targeting superoxide-mediated wnt signaling pathways. American Journal of the Medical Sciences $\mathbf{3 5 1}$ 286-295. (https://doi.org/10.1016/j.amjms.2015.12.017)

Jackson KM, DeLeon M, Verret CR \& Harris WB 2002 Dibenzoylmethane induces cell cycle deregulation in human prostate cancer cells. Cancer Letters 178 161-165. (https://doi.org/10.1016/S03043835(01)00844-8)

Jeong HW, Hsu KC, Lee JW, Ham M, Huh JY, Shin HJ, Kim WS \& Kim JB 2009 Berberine suppresses proinflammatory responses through AMPK activation in macrophages. American Journal of Physiology-Endocrinology and Metabolism 296 E955-E964. (https://doi. org/10.1152/ajpendo.90599.2008)

Keaney JF Jr, Larson MG, Vasan RS, Wilson PW, Lipinska I, Corey D, Massaro JM, Sutherland P, Vita JA, Benjamin EJ, et al. 2003 Obesity and systemic oxidative stress: clinical correlates of oxidative stress in the Framingham Study. Arteriosclerosis, Thrombosis, and Vascular Biology 23 434-439. (https://doi.org/10.1161/01 ATV.0000058402.34138.11)

Khor TO, Yu S, Barve A, Hao X, Hong JL, Lin W, Foster B, Huang MT, Newmark HL \& Kong AN 2009 Dietary feeding of dibenzoylmethane inhibits prostate cancer in transgenic adenocarcinoma of the mouse prostate model. Cancer Research 69 7096-7102. (https://doi. org/10.1158/0008-5472.CAN-09-0597)

Kim J, Kwak HJ, Cha JY, Jeong YS, Rhee SD, Kim KR \& Cheon HG 2014 Metformin suppresseslipopolysaccharide (LPS)-induced inflammatory response in murine macrophages via activating transcription factor-3 (ATF-3) induction. Journal of Biological Chemistry 289 23246-23255. (https://doi.org/10.1074/jbc.M114.577908)

Kim N, Kim HM, Lee ES, Lee JO, Lee HJ, Lee SK, Moon JW, Kim JH, Kim JK, Kim SJ, et al. 2015 Dibenzoylmethane exerts metabolic activity through regulation of AMP-activated protein kinase (AMPK)mediated glucose uptake and adipogenesis pathways. PLOS ONE 10 e0120104. (https://doi.org/10.1371/journal.pone.0120104)

Kim BH, Lee ES, Choi R, Nawaboot J, Lee MY, Lee EY, Kim HS \& Chung CH 2016 Protective effects of curcumin on renal oxidative stress and lipid metabolism in a rat model of type 2diabetic nephropathy. Yonsei Medical Journal 57 664-673. (https://doi. org/10.3349/ymj.2016.57.3.664)

Kodera R, Shikata K, Takatsuka T, Oda K, Miyamoto S, Kajitani N, Hirota D, Ono T, Usui HK \& Makino H 2014 Dipeptidyl peptidase-4 inhibitor ameliorates early renal injury through its anti-inflammatory action in a rat model of type 1 diabetes. Biochemical and Biophysical Research Communications 443 828-833. (https://doi.org/10.1016/j. bbrc.2013.12.049)

Lu M, Yin N, Liu W, Cui X, Chen S \& Wang E 2017 Curcumin ameliorates diabetic nephropathy by suppressing NLRP3 inflammasome signaling. BioMed Research International 2017 1516985. (https://doi. org/10.1155/2017/1516985)

Maithilikarpagaselvi N, Sridhar MG, Swaminathan RP \& Sripradha R 2016 Preventive effect of curcumin on inflammation, oxidative stress and insulin resistance in high-fat fed obese rats. Journal of Complementary and Integrative Medicine 13 137-143. (https://doi.org/10.1515/jcim2015-0070 )

McArdle MA, Finucane OM, Connaughton RM, McMorrow AM \& Roche HM 2013 Mechanisms of obesity-induced inflammation and insulin resistance: insights into the emerging role of nutritional strategies. Frontiers in Endocrinology 4 52. (https://doi.org/10.3389/ fendo.2013.00052)

Mezzano S, Aros C, Droguett A, Burgos ME, Ardiles L, Flores C, Schneider H, Ruiz-Ortega M \& Egido J 2004 NF-kappaB activation and overexpression of regulated genes in human diabetic nephropathy. https://joe.bioscientifica.com https://doi.org/10.1530/JOE-18-0206
(C) 2019 Society for Endocrinology Published by Bioscientifica Ltd. Printed in Great Britain 
Nephrology Dialysis Transplantation 19 2505-2512. (https://doi. org/10.1093/ndt/gfh207)

Miller EJ, Li J, Leng L, McDonald C, Atsumi T, Bucala R \& Young LH 2008 Macrophage migration inhibitory factor stimulates AMP-activated protein kinase in the ischaemic heart. Nature $\mathbf{4 5 1} 578-582$. (https:// doi.org/10.1038/nature06504)

Nguyen D, Ping F, Mu W, Hill P, Atkins RC \& Chadban SJ 2006 Macrophage accumulation in human progressive diabetic nephropathy. Nephrology 11 226-231. (https://doi.org/10.1111/j.14401797.2006.00576.x)

Nistala R, Habibi J, Lastra G, Manrique C, Aroor AR, Hayden MR, Garro M, Meuth A, Johnson M, Whaley-Connell A, et al. 2014 Prevention of obesity-induced renal injury in male mice by DPP4 inhibition. Endocrinology 155 2266-2276. (https://doi.org/10.1210/ en.2013-1920)

Pan Y, Zhang X, Wang Y, Cai L, Ren L, Tang L, Wang J, Zhao Y, Wang Y, Liu Q, et al. 2013a Targeting JNK by a new curcumin analog to inhibit NF-kB-mediated expression of cell adhesion molecules attenuates renal macrophage infiltration and injury in diabetic mice. PLOS ONE 8 e79084. (https://doi.org/10.1371/journal.pone.0079084)

Pan Y, Zhu G, Wang Y, Cai L, Cai Y, Hu J, Li Y, Yan Y, Wang Z, Li X, et al. $2013 b$ Attenuation of high-glucose-induced inflammatory response by a novel curcumin derivative B06 contributes to its protection from diabetic pathogenic changes in rat kidney and heart. Journal of Nutritional Biochemistry 24 146-155. (https://doi.org/10.1016/j. jnutbio.2012.03.012)

Qatanani M \& Lazar MA 2007 Mechanisms of obesity-associated insulin resistance: many choices on the menu. Genes and Development $\mathbf{2 1}$ 1443-1455. (https://doi.org/10.1101/gad.1550907)

Sassy-Prigent C, Heudes D, Mandet C, Belair MF, Michel O, Perdereau B, Bariety J \& Bruneval P 2000 Early glomerular macrophage recruitment in streptozotocin-induced diabetic rats. Diabetes 49 466-475. (https:// doi.org/10.2337/diabetes.49.3.466)

Sedeek M, Callera G, Montezano A, Gutsol A, Heitz F, Szyndralewiez C, Page P, Kennedy CR, Burns KD, Touyz RM, et al. 2010 Critical role of Nox4-based NADPH oxidase in glucose-induced oxidative stress in the kidney: implications in type 2 diabetic nephropathy. American Journal of Physiology-Renal Physiology 299 F1348-F1358. (https://doi. org/10.1152/ajprenal.00028.2010)

Seok SJ, Lee ES, Kim GT, Hyun M, Lee JH, Chen S, Choi R, Kim HM, Lee EY \& Chung CH 2013 Blockade of CCL2/CCR2 signalling ameliorates diabetic nephropathy in $\mathrm{db} / \mathrm{db}$ mice. Nephrology Dialysis Transplantation 28 1700-1710. (https://doi.org/10.1093/ndt/gfs555)

Serrano Rios M 1998 Relationship between obesity and the increased risk of major complications in non-insulin-dependent diabetes mellitus. European Journal of Clinical Investigation 28 (Supplement 2) 14-17, discussion 17-18. (https://doi.org/10.1046/j.13652362.1998.0280s2014.x)

Shishu, Singla AK \& Kaur IP 2003 Inhibitory effect of dibenzoylmethane on mutagenicity of food-derived heterocyclic amine mutagens. Phytomedicine 10 575-582. (https://doi. org/10.1078/094471103322331575)

Soetikno V, Sari FR, Veeraveedu PT, Thandavarayan RA, Harima M, Sukumaran V, Lakshmanan AP, Suzuki K, Kawachi H \& Watanabe K 2011 Curcumin ameliorates macrophage infiltration by inhibiting NF-kappaB activation and proinflammatory cytokines in streptozotocin induced-diabetic nephropathy. Nutrition and Metabolism 8 35. (https://doi.org/10.1186/1743-7075-8-35)

Soetikno V, Sari FR, Sukumaran V, Lakshmanan AP, Harima M, Suzuki K, Kawachi H \& Watanabe K 2013 Curcumin decreases renal triglyceride accumulation through AMPK-SREBP signaling pathway in streptozotocin-induced type 1 diabetic rats. Journal of Nutritional Biochemistry 24 796-802. (https://doi.org/10.1016/j. jnutbio.2012.04.013)

Sun K, Kusminski CM \& Scherer PE 2011 Adipose tissue remodeling and obesity. Journal of Clinical Investigation 121 2094-2101. (https://doi. org/10.1172/JCI45887)

Takano K, Kitao Y, Tabata Y, Miura H, Sato K, Takuma K, Yamada K, Hibino S, Choshi T, Iinuma M, et al. 2007 A dibenzoylmethane derivative protects dopaminergic neurons against both oxidative stress and endoplasmic reticulum stress. American Journal of Physiology-Cell Physiology 293 C1884-C1894. (https://doi.org/10.1152/ ajpcell.00305.2007)

Trevisan R, Dodesini AR \& Lepore G 2006 Lipids and renal disease. Journal of the American Society of Nephrology 17 S145-S147. (https://doi org/10.1681/ASN.2005121320)

Trujillo J, Chirino YI, Molina-Jijon E, Anderica-Romero AC, Tapia E \& Pedraza-Chaverri J 2013 Renoprotective effect of the antioxidant curcumin: recent findings. Redox Biology 1 448-456. (https://doi. org/10.1016/j.redox.2013.09.003)

Wang Y, Chang J, Yao B, Niu A, Kelly E, Breeggemann MC, Abboud Werner SL, Harris RC \& Zhang MZ 2015 Proximal tubule-derived colony stimulating factor-1 mediates polarization of renal macrophages and dendritic cells, and recovery in acute kidney injury. Kidney International 88 1274-1282. (https://doi.org/10.1038/ki.2015.295)

Wu H, Kong L, Tan Y, Epstein PN, Zeng J, Gu J, Liang G, Kong M, Chen X, Miao L, et al. 2016 C66 ameliorates diabetic nephropathy in mice by both upregulating NRF2 function via increase in miR-200a and inhibiting miR-21. Diabetologia 59 1558-1568. (https://doi. org/10.1007/s00125-016-3958-8)

Xiong ZE, Dong WG, Wang BY, Tong QY \& Li ZY 2015 Curcumin attenuates chronic ethanol-induced liver injury by inhibition of oxidative stress via mitogen-activated protein kinase/nuclear factor E2-related factor 2 pathway in mice. Pharmacognosy Magazine 11 707-715. (https://doi.org/10.4103/0973-1296.165556)

Yao F, Zhang M \& Chen L 2016 5'-Monophosphate-activated protein kinase (AMPK) improves autophagic activity in diabetes and diabetic complications. Acta Pharmaceutica Sinica B 6 20-25. (https://doi. org/10.1016/j.apsb.2015.07.009)

You YH, Okada S, Ly S, Jandeleit-Dahm K, Barit D, Namikoshi T \& Sharma K 2013 Role of Nox2 in diabetic kidney disease. American Journal of Physiology-Renal Physiology 304 F840-F848. (https://doi. org/10.1152/ajprenal.00511.2012)

Zhang X, Chen M, Zou P, Kanchana K, Weng Q, Chen W, Zhong P, Ji J, Zhou H, He L, et al. 2015 Curcumin analog WZ35 induced cell death via ROS-dependent ER stress and G2/M cell cycle arrest in human prostate cancer cells. BMC Cancer 15 866. (https://doi.org/10.1186/ s12885-015-1851-3)

Zhao NJ, Liao MJ, Wu JJ \& Chu KX 2018 Curcumin suppresses Notch1 signaling: improvements in fatty liver and insulin resistance in rats. Molecular Medicine Reports 17 819-826. (https://doi.org/10.3892/ mmr.2017.798)

Received in final form 16 October 2018

Accepted 31 October 2018 https://joe.bioscientifica.com https://doi.org/10.1530/JOE-18-0206
(C) 2019 Society for Endocrinology Published by Bioscientifica Ltd.
Printed in Great Britain 